\title{
Implantação do Business Intelligence (BI) em um Grupo de Concessionárias do Interior de Minas Gerais
}

\author{
Business Intelligence (BI) Deployment in a Dealership Group in the Countryside of \\ Minas Gerais
}

\author{
Zandra Cristina Lima Silva Queiroz ${ }^{1}$ \\ Giselle Alves Mundim Costa ${ }^{2}$
}

\begin{abstract}
Resumo
Dentre as aplicações tecnológicas que tratam o grande volume de informações para tomada de decisão, destaca-se o uso do Business Intelligence (BI), o qual permite a identificação de padrões de comportamento e ocorrências de eventos que agregam valor e conhecimento para as empresas. Entretanto, para que o BI possa proporcionar tais benefícios, o alinhamento entre a Tecnologia da Informação (TI) e o negócio é fundamental para o sucesso da implantação dessa ferramenta. Neste contexto, o presente estudo tem por objetivo apresentar a implantação do sistema BI em uma concessionária de veículos no interior de Minas Gerais a partir do diagnóstico do nível de alinhamento entre a TI e o negócio. Como metodologia foi realizada uma pesquisa descritiva, aplicada com abordagem mista. Para o diagnóstico do nível de alinhamento foi utilizada a metodologia de Lima (2008) por meio de questionários. Nas etapas posteriores da implantação do BI na organização foi utilizada a metodologia de Bezerra e Siebra (2015). Os resultados do estudo demonstram que a aplicação do modelo de diagnóstico do nível de alinhamento entre a TI e o negócio possibilitou a identificação de oportunidades de melhoria que promoveu a implantação do BI, cuja contribuição é a evidenciação de um caso de sua implantação em empresas do ramo de concessionárias de veículos do interior de Minas Gerais.

Palavras-Chave: Business Intelligence. Controladoria. Tecnologia da Informação. Alinhamento Estratégico.
\end{abstract}

\begin{abstract}
Among the technological applications that deal with the large volume of information for decision making, the use of Business Intelligence (BI) stands out, which allows the identification of behavior patterns and events occurrences that add value and knowledge to companies. However, for BI to provide such benefits, the alignment between Information Technology (IT) and the business is essential for the successful implementation of this tool. In this context, this study aims to present the implantation of the BI system in a car dealership in the countryside of Minas Gerais considering the diagnosis of the level of alignment between IT and the business. As a methodology, a descriptive research was carried out, applied with a mixed approach. For the diagnosis of the level of alignment, the methodology of Lima (2008) was used through questionnaires. In the later stages of the BI implementation in the
\end{abstract}

\footnotetext{
${ }^{1}$ Universidade Federal de Uberlândia - UFU, Brasil. ORCID https://orcid.org/0000-00033362-8681. E-mail: zandraqueiroz@gmail.com

2 Universidade Federal de Uberlândia - UFU, Brasil. ORCID https://orcid.org/0000-00017471-336X. E-mail: cmgiselle@icloud.com
} 
organization, the Bezerra and Siebra (2015) methodology was used. The results of the study demonstrate that the application of the diagnostic model of the level of alignment between IT and the business enabled the identification of improvement opportunities that promoted the implantation of $\mathrm{BI}$, whose contribution is the evidence of a case of its implantation in companies of the branch of vehicle dealerships in the countryside of Minas Gerais.

Keywords: Business Intelligence. Controllership. Information Technology. Strategic Alignment.

$$
* * *
$$

\section{Introdução}

O grande volume de informações produzido nos últimos anos, em decorrência dos avanços tecnológicos, impõe às empresas a adoção de estratégias de gestão para viabilizar o uso de todos os recursos e informações disponíveis. As ferramentas tecnológicas, segundo Fátima e Linnes (2019), são conhecidas como sistemas de inteligência de negócios. Segundo os autores, referem-se ao uso de ferramentas, aplicativos e melhores práticas que auxiliam o tratamento das informações produzidas pelos sistemas de informação e são fundamentais para que as empresas possam analisá-las e utilizá-las de forma ágil e assertiva no processo de tomada de decisão.

Os sistemas de inteligência de negócios tiveram sua origem, segundo Kenneth e Laudon (2011), com os Sistemas de Apoio à Decisão (SAD), que se caracterizavam como um tipo de sistema de informação que auxiliava o corpo gerencial das organizações no processo da tomada de decisão.

Desde então, os SAD evoluíram para o conceito de Business Intelligence (BI), que de acordo com alguns autores, proporcionou um avanço na análise e no diagnóstico do ambiente e dos processos, permitindo às organizações e seus gestores melhores condições de antecipar o futuro e reduzir riscos e incertezas na tomada de decisão (Silva, Silva \& Gomes, 2016; Watson \& Wixon, 2007). Sendo assim, o termo BI tem sido amplamente utilizado como conceito de sistemas baseados em tecnologia de informação para suporte à tomada de decisão. Pinto (2012) estudou algumas definições de BI encontradas na literatura e as redefiniu como um conjunto 
de metodologias de gestão que possui uma estrutura dedicada à coleta de dados e à conversão desses em informações e conhecimentos por meio de um processo organizado e sistematizado que permite às organizações adquirir, analisar e distribuir informações para alavancar processos e conduzi-la a melhores decisões e ações a fim de melhorar o desempenho da empresa.

Diante disso, Silva, Silva e Gomes (2016) verificaram que para implementar um BI, além de compreender a origem das informações, também é necessário conhecer os processos organizacionais e como esses podem influenciar na tomada de decisão. Os autores afirmam ainda que tais informações são levantadas junto à equipe de gestão da organização e complementam ao dizer que "faz-se necessário que os gestores forneçam informações corretas e alinhadas aos objetivos estratégicos a fim de facilitar os ajustes necessários e permitir a implantação e aplicação do BI para obtenção do sucesso" (Silva, Silva \& Gomes, 2016, p. 2795).

Guarda et al (2016) acrescenta que além de informações internas, as empresas também devem considerar informações do ambiente externo tais como clientes, concorrentes e mercado. Nesse sentido, Fátima e Linnes (2019) também verificaram que para garantir a implementação bemsucedida de um BI é fundamental identificar os fatores críticos do sucesso das organizações. Em seu estudo, os autores analisaram publicações de 2010 a 2018 sobre tais fatores e encontraram como principais resultados: falta de apoio da alta gestão, necessidade de uma definição clara da visão do negócio para a sua correta implantação, necessidade de metodologia orientada para o negócio e gestão de projetos, participação ativa dos usuários na construção do BI, equipe qualificada e com conhecimentos técnicos e do negócio, informações com qualidade, integridade e infraestrutura escalável, que dependerão, segundo os autores, da eficácia do planejamento, implementação e adoção do BI (Fátima \& Linnes, 2019).

Para isso, é fundamental a integração das áreas de Controladoria, Tecnologia da Informação e demais áreas da empresa. Nesse sentido, existem estudos que constataram que o alinhamento entre a TI e o negócio 
proporciona uma melhoria quanto ao uso da tecnologia no processo da tomada de decisão. Affeldt, Silveira e Vanti (2006) observou que tal alinhamento vai além do apoio operacional aos processos do negócio, que ele influencia a gestão do negócio através das informações e análises que proporciona. Joia e Souza (2009) observaram que, por meio do alinhamento, os profissionais da TI têm a oportunidade de se inteirar sobre os negócios da organização e contribuir para a aplicação de novas tecnologias e os gestores a oportunidade de conhecer o papel da tecnologia da informação e as suas possibilidades de uso. Amarilli, Van Vliet e Van Den Hooff (2017) também observou a existência de vínculos entre alinhamento e desempenho, demonstrados em vários estudos.

$\mathrm{Na}$ Controladoria também foram encontrados estudos que identificaram que o BI auxilia o departamento ao disponibilizar informações confiáveis para o processo decisório e que representa um dos principais instrumentos no cenário dos negócios por proporcionar informações operacionais, gerenciais e estratégicas (Affeldt et al 2006; Reginato \& Nascimento, 2007).

Bezerra e Siebra (2015) também observaram que para a implantação de um BI é importante um planejamento adequado de acordo com os objetivos da organização. Além disso, Fátima e Linnes (2019) observaram a importância de estudos de caso que demonstrem o uso bem-sucedido do BI em pequenas e médias empresas para que outras possam aprender com o seu sucesso. Neste contexto, entendendo a importância do uso de informações estratégicas para tomada de decisão e que os sistemas de BI são tecnologias capazes de fornecê-las, além de contribuir com o alinhamento entre TI e o negócio, tem-se a seguinte questão de pesquisa: como se dá a implantação de um sistema de BI uma concessionária de veículos do interior de Minas Gerais?

Para responder à questão, este trabalho tem por objetivo demonstrar a implantação de um sistema de BI em um grupo de concessionárias no interior de Minas Gerais a partir do diagnóstico do nível de alinhamento 
entre a TI e o negócio. O estudo é justificado por demonstrar a implantação do BI em uma empresa e contribuir com a literatura ao apontar casos de uso da tecnologia para análise de informações em uma organização. Além disso o trabalho demonstra o uso de análise do nível de maturidade do alinhamento entre TI e o negócio, como forma de identificar uma melhoria no alinhamento entre a TI e a estratégia da organização por meio do uso do BI.

O estudo foi realizado em uma concessionária de veículos por considerar a relevância deste setor no cenário comercial brasileiro, visto que responde pela geração de 315 mil empregos diretos, correspondendo a 5,12 \% do Produto Interno Bruto (PIB) Federação Nacional da Distribuição de Veículos Automotores [FENABRAVE] (2021).

$\mathrm{O}$ artigo encontra-se estruturado em quatro secções além desta. $\mathrm{Na}$ segunda seção será apresentado o referencial teórico. $\mathrm{Na}$ terceira seção a metodologia utilizada. Na quarta seção a descrição dos resultados da implantação do sistema BI e na quinta e última seção as considerações finais do trabalho.

\section{Referencial Teórico}

\subsection{Alinhamento da TI e o Negócio}

Luftman (2004, p. 100) conceitua o alinhamento entre a TI e o negócio como "a aplicação da Tecnologia da Informação (TI) de forma adequada e oportuna em harmonia com as estratégias, objetivos e necessidades das organizações”. Neste sentido, o autor infere que o processo de alinhamento é dinâmico e envolve a correta harmonização das necessidades do negócio. Ele completa ao ressaltar como a TI poderá atendê-las no tempo certo.

O conceito de alinhamento é derivado do alinhamento estratégico e tem como princípio as noções clássicas de estratégia que abordam o conjunto de decisões para o alcance dos objetivos específicos da organização. Silveira (2017), ao traçar um histórico do conceito de alinhamento estratégico, observou que ele é aplicado a várias áreas do conhecimento e que se refere ao alinhamento das ações e práticas que envolvem a configuração da 
empresa, à interação e gestão de complexas coligações entre pessoas, processos e tecnologias, resultantes das relações entre gestores, funcionários, fornecedores, instituições educacionais, associações profissionais, infraestrutura e muito mais. O alinhamento estratégico, por sua vez, deriva do conceito de estratégia que significa segundo Johnson, Scholes e Whittington (2011, p. 25) a "orientação e o alcance de uma organização, em longo prazo, que conquista vantagens num ambiente inconstante por meio da configuração de recursos e competências com o intuito de atender as expectativas dos stakeholders".

Esses conceitos, também aplicados à TI, são referências às ações da empresa e podem se tornar uma vantagem competitiva em relação aos concorrentes, fornecedores, produtos substitutos, novos entrantes no mercado, visto que, segundo Kenneth e Laudon (2011), tais vantagens competitivas, na maioria das vezes, são obtidas por meio do uso dos sistemas de informação em conjunto com todo o arsenal tecnológico como redes, bancos de dados, Internet, entre outros.

Dessa forma, para que as empresas possam definir as suas estratégias, a área de negócios deve estar alinhada à sua estrutura de TI e ao que ela oferece. Além disso, a área de TI deve estar apta a entender as demandas da empresa e buscar soluções que proporcionem a melhor execução de suas estratégias. Nesta perspectiva, Amarilli et al. (2017) observaram que o alinhamento da TI aos negócios de uma empresa é um tópico amplamente estudado, uma vez que possui diversas pesquisas que demonstram a grande relação entre o alinhamento e o desempenho corporativo. Em contrapartida, os autores observam a carência de estudos que traduzam as práticas aplicadas no dia a dia das empresas.

Existem autores que discutem maneiras de se obter o alinhamento correto e como alcançá-lo. Luftman (2004) constatou que perguntas importantes precisam ser abordadas: como as organizações podem avaliar o alinhamento? Como as organizações podem melhorar o alinhamento? Como as organizações podem alcançar um alinhamento maduro? Diante de tais 
questionamentos, o autor demonstrou um modelo de avaliação da maturidade do alinhamento da TI e dos negócios em cinco níveis.

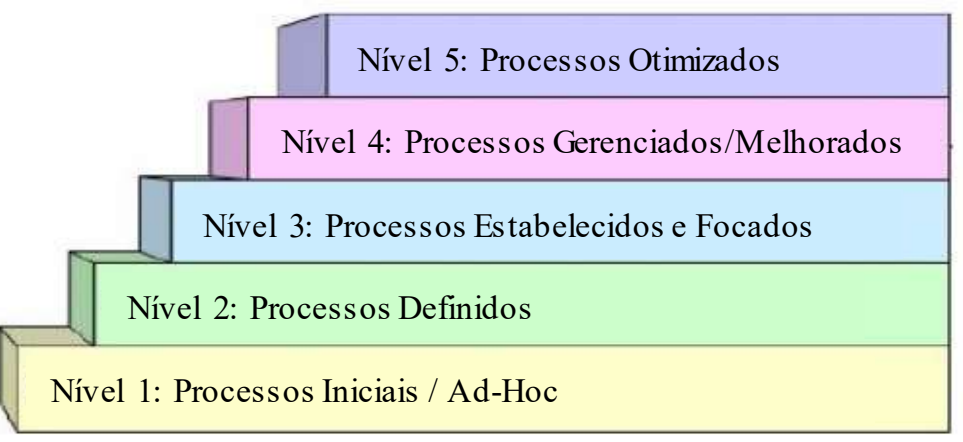

Figura 1. Níveis de maturidade do alinhamento estratégico Fonte: Luftman (2000, p. 10, apud Lima, 2008, p. 65).

Lima (2008) também estudou cada um dos cinco níveis de maturidade do alinhamento que se concentram, por sua vez, um conjunto de seis critérios: Maturidade das Comunicações, Maturidade da Competência / Medição de Valor, Maturidade da Governança, Maturidade da Parceria, Escopo e Maturidade da Arquitetura, Maturidade das Competências.

De acordo com o autor, por meio deste modelo, é possível conhecer o nível de maturidade do alinhamento da TI, entender como a empresa enxerga essa área e como ela poderá contribuir para o sucesso da empresa. Luftman (2004, p.101) também afirma que "identificar a maturidade do alinhamento de uma organização é um excelente veículo para compreender e melhorar o relacionamento entre os negócios e a TI”. Em outro estudo, Luftman, Lyytinen e Zvi (2017) demonstraram um modelo de avaliação de um exemplo de sucesso, o qual demonstrou que, por meio do compartilhamento de atividades entre as áreas funcionais e a TI, as empresas estudadas obtiveram um melhor alinhamento. Dentre as ações, observaram que quando as áreas de finanças, marketing e $\mathrm{RH}$ realizaram o planejamento e a coordenação de metas em conjunto com a TI, obtiveram um nível maior de alinhamento e por consequência um impacto positivo no desempenho da empresa. 
Lima (2008) também constatou que no processo de avaliação para detecção do nível de maturidade do alinhamento estratégico de uma organização, o autor usou o modelo de Luftman, e observou que os gaps gerados pela análise das respostas são convertidos em oportunidades de melhorias que consolidam cada vez mais tal alinhamento.

Neste sentido, Luftman et al (2017) também pesquisaram que um alinhamento bem-sucedido entre a TI e o negócio gera impacto positivo na melhoria do desempenho da organização pois promove uma maior interação das atividades que gerentes da TI e de negócios precisam trabalhar em conjunto para coordenar metas e operações, tanto dentro da TI quanto em outras funções organizacionais (por exemplo: finanças, marketing, $\mathrm{RH}$ ). Os autores observaram que "A avaliação do status de alinhamento de uma empresa é uma etapa fundamental na identificação de ações necessárias para melhorar a relação congruente entre negócios e TI e para garantir que a TI está sendo aproveitada para fornecer valor aos negócios" (Luftman et al 2017, p. 12). Sendo assim, a implantação do BI configura-se em uma atividade que demonstra a evidência da busca por melhoria no uso da TI pelas organizações por meio do alinhamento entre a área da TI e o negócio.

\subsection{Ferramentas de BI}

A Tecnologia da Informação e os seus respectivos recursos de geração e transmissão da informação proveem meios para a obtenção das informações necessárias para a Controladoria (Rezende e Abreu 2013). Neste sentido, as ferramentas do Business Intelligence (BI) ampliam a função dos sistemas de informação fornecendo uma visão sistêmica do negócio e ajudam na distribuição uniforme dos dados entre os usuários, ou seja, transformam grandes quantidades de dados em informações de qualidade para a tomada de decisões (Barbieri, 2011). Guarda et al (2016) acrescentam que as ferramentas do BI deverão oferecer além das informações internas da organização, informações estratégicas de mercado que são essenciais para o sucesso do negócio. 
Alguns autores afirmam que o BI atua como um "guarda-chuva" que une as informações de vários ambientes, utilizando-as de várias formas de acordo com a necessidade do negócio (Bezerra e Siebra 2015; Silva, Silva e Gomes 2016). Além disso, também permite a identificação de padrões de comportamento e ocorrências de eventos que agregam valor e conhecimento para as empresas e viabilizam o uso de conceitos estatísticos, matemáticos e inteligência artificial (Silva, Silva e Gomes 2016; Moreira et al, 2016).

Dentre os benefícios de sua utilização, estudos apontam que o uso do BI contribui para o controle e acompanhamento das atividades, bem como fornece uma visão sistêmica do negócio por meio dos indicadores de desempenho. Nesse sentido, Reginato e Nascimento (2007) verificaram que o uso do BI auxiliou a Controladoria no provimento de informações confiáveis, proporcionando à empresa estudada a melhoria dos resultados.

Sharda, Delen e Turban (2019, p.15) destacam outro benefício do BI: a visualização dos resultados por meio de dashboard, que são "exibições visuais que possibilitam uma clara compreensão dos dados, além de incorporarem capacidades de aprofundamento e revisão detalhada dos dados com menos esforço e em menos tempo". Nesse sentido, alguns estudos atestam a aplicação dos dashboards para a visualização de painéis dos indicadores de desempenho (Serra Costa e Duarte 2010; Fraga, Erpen, Varvakis e Santos 2017; Strate e Pretto 2015).

Bezerra e Siebra (2015) enfatizam que para garantir os benefícios do uso do BI, a sua implantação deverá ser realizada de forma organizada desde a sua preparação até o tratamento dos dados. Os autores propõem a construção do BI em etapas baseada no Planejamento Estratégico Corporativo (PEC) e no Planejamento Estratégico da Informação (PEI). O PEC fornecerá os indicadores de desempenho e as metas organizacionais e o PEI guiará quanto as fontes de obtenção das informações para a construção do BI. A Figura 2 apresenta as etapas propostas pelos autores. 


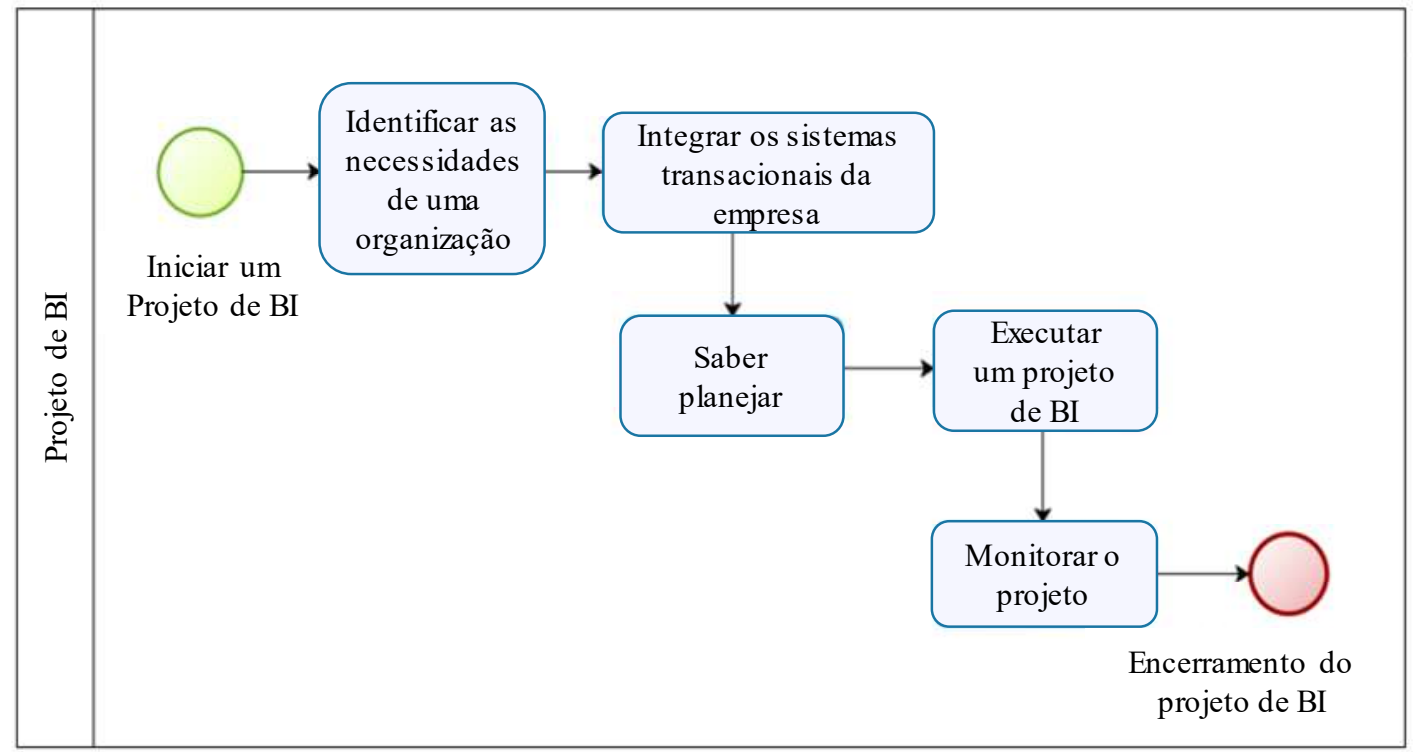

Figura 2. Modelo projeto implantação de BI Fonte: Bezerra e Siebra (2015, p. 236).

As etapas propostas, segundo os autores, devem partir da identificação dos objetivos da organização e dos respectivos indicadores. Em seguida são levantadas as fontes dos dados que compõem tais indicadores dos sistemas utilizados pela empresa. Posteriormente, são criados planos de ação que irão nortear a implantação do BI de acordo com os objetivos definidos.

\section{Procedimentos Metodológicos}

Como o principal objetivo deste trabalho é demonstrar a implantação de um sistema de BI em uma concessionária de veículos no interior de Minas Gerais, esta pesquisa caracteriza-se por ser um estudo descritivo. Segundo Gil (2010), esta tem como principal objetivo descrever as características de uma população ou fenômeno.

Quanto a sua natureza, esta é classificada como empírica aplicada pois contribui com a apresentação da implantação de um sistema de BI em uma organização. De acordo com Demo (2009) este tipo de pesquisa é caracterizado pela produção e análise de dados que representam a realidade dos fatos e como a teoria é aplicada na prática. 
A abordagem da pesquisa é mista. $\mathrm{Na}$ abordagem quantitativa foram utilizados instrumentos estatísticos para analisar o nível de maturidade do alinhamento estratégico entre a TI e o negócio. A abordagem qualitativa foi usada para identificar oportunidades de melhorias que proporcionem um aumento do nível de maturidade e comprovar a utilização de modelos de avaliação da maturidade com foco na melhoria do alinhamento entre a TI e a estratégia da organização.

A coleta de dados foi realizada por meio de questionário aplicado em 17 gestores da empresa no período de janeiro a março de 2016. Este questionário baseou-se no modelo proposto por Lima (2008) composto por 60 perguntas distribuídas em seis blocos por atributo e foi enviado para todos os gestores da empresa e as respostas foram tabuladas por meio de planilhas.

Para calcular o nível de maturidade por atributo e geral, foi utilizada a medida moda para cada questão. Adicionalmente, foi realizada pesquisa documental a fim de confrontar os resultados obtidos do nível de alinhamento da TI, por atributo e no geral, com os objetivos da empresa, coletados em relatórios de planejamento de cada setor. O objetivo foi apurar as oportunidades de melhoria.

Para demonstrar a implantação de um sistema de BI a partir do diagnóstico do nível de alinhamento entre a TI e o negócio de uma concessionária de veículos, o desenvolvimento do presente estudo utilizou a metodologia proposta por Lima (2008) na Etapa 1: quando foram levantados os dados para medir o nível de maturidade por atributo e no geral, assim como na Etapa 2: quando foram analisados os resultados obtidos. Na Etapa 1 , confrontando os valores de maturidade obtidos por critério versus valor geral, foi gerada a construção do relatório de oportunidades de melhoria do aumento do nível de alinhamento.

A Etapa 3 corresponde à demonstração do objeto desta pesquisa, que apresenta o plano de implantação do BI como uma das oportunidades de melhoria para o aumento do nível de alinhamento. Nesta etapa, foi utilizado 
o modelo de implantação do BI proposto por Bezerra e Siebra (2015). A

Tabela 1, a seguir, descreve cada passo da metodologia utilizada.

Tabela 1. Etapas de implantação do BI

\begin{tabular}{|c|c|}
\hline Etapas & Descrição de atividades \\
\hline \multicolumn{2}{|l|}{1 - Avaliação Alinhamento da TI } \\
\hline 1 - Preparação e diagnóstico & $\begin{array}{l}\text { Identificar o perfil da organização, os níveis } \\
\text { decisórios e a equipe. }\end{array}$ \\
\hline 2 - Coleta de documentos & $\begin{array}{l}\text { Identificar a estratégia, a organização da } \\
\text { empresa, métricas e responsabilidades da TI. }\end{array}$ \\
\hline $\begin{array}{l}3 \text { - Análise do questionário e apresentação do } \\
\text { resultado }\end{array}$ & $\begin{array}{l}\text { Aplicação do questionário, consolidação dos } \\
\text { dados e a medida do nível de maturidade. }\end{array}$ \\
\hline 2 - Levantamento das oportunidades de melhoria & $\begin{array}{l}\text { Analisar a harmonia entre os valores de } \\
\text { maturidade obtidos por atributo versus valor } \\
\text { geral. }\end{array}$ \\
\hline \multicolumn{2}{|l|}{3 - Implantação do BI } \\
\hline \multicolumn{2}{|c|}{$\begin{array}{l}1 \text { - Definição dos indicadores e a descrição das fontes Identificar as reais necessidades da empresa. } \\
\text { dos dados }\end{array}$} \\
\hline 2 - Estruturação da base de dados & $\begin{array}{l}\text { Integrar todos os sistemas transacionais da } \\
\text { empresa. }\end{array}$ \\
\hline $\begin{array}{l}3 \text { - Elaboração dos relatórios dos indicadores } \\
4 \text { - Construção do BI }\end{array}$ & Planejamento, execução e controle. \\
\hline
\end{tabular}
Fonte: adaptado de Lima (2008).

A execução das etapas descritas na metodologia foi realizada pela equipe de TI e Controladoria da organização em estudo que realizaram a documentação e registro de cada atividade realizada para realização da análise dos dados coletados.

\section{Apresentação e Análise dos Resultados}

\subsection{Análise do Alinhamento Estratégico entre a TI e o Negócio}

A análise do nível de maturidade geral demonstrou que a empresa está no nível três (3) de alinhamento estratégico entre a TI e o negócio, ou seja, os processos estão estabelecidos com boa comunicação entre as áreas envolvidas e em alguns atributos as métricas já estão implementadas com eficiência no nível quatro (4) e cinco (5).

Quanto à parceria da área da TI com as outras áreas da empresa, essa está no nível cinco (5), demonstrando que a área da TI é reconhecida e 
vista como recurso impulsionador dos processos do negócio com tecnologias integradas e equipe qualificada.

O atributo Governança da TI já está estabelecido na empresa no nível quatro (4) de maturidade, o que indica, segundo Lima (2008), o reconhecimento da TI no nível estratégico da organização.

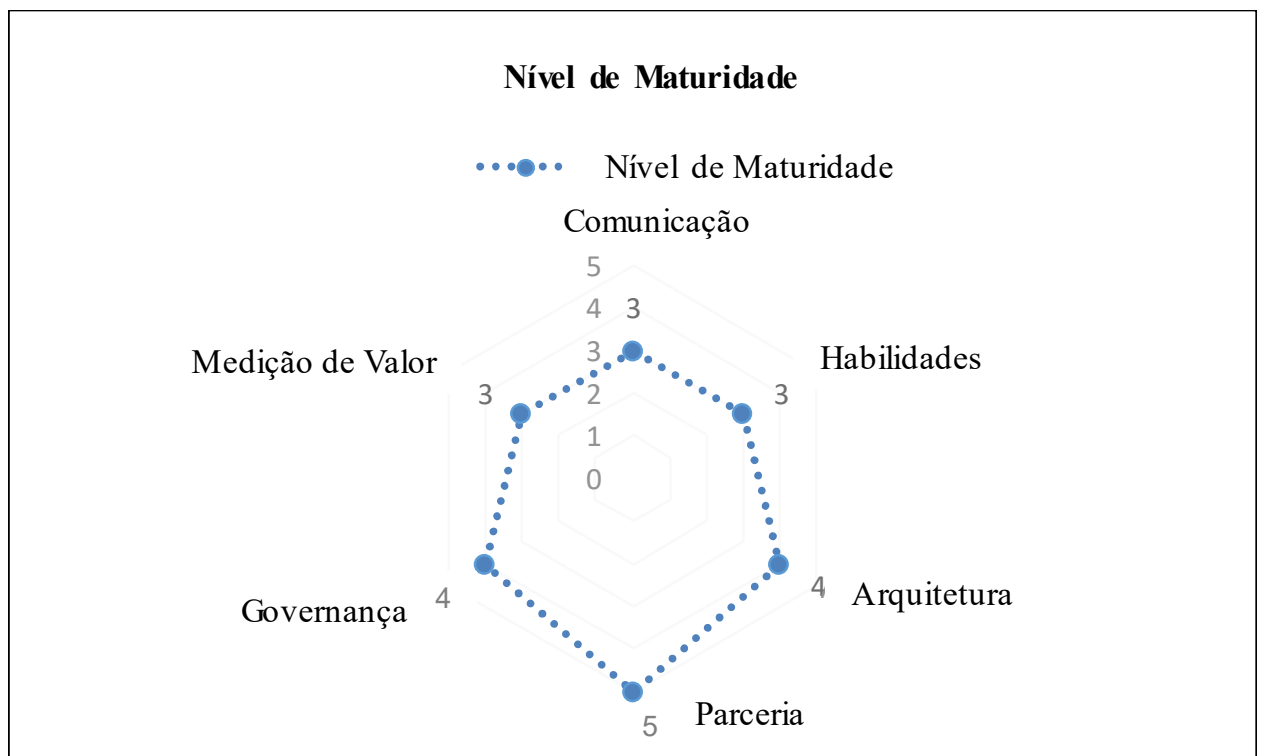

Figura 3. Resultado da análise do alinhamento estratégico entre a TI e o negócio Fonte: do próprio autor.

Os atributos habilidades, comunicação e medição de valor obtiveram o mesmo resultado três (3). Eles indicam que as métricas da TI apresentam dificuldades para apoiar estrategicamente as áreas da organização. Tais resultados, segundo Lima (2008), devem ser confrontados com os dados coletados das mesmas áreas a fim de identificar as oportunidades de melhoria do nível de maturidade.

\subsection{Levantamento de Oportunidades de Melhoria}

Nos atributos que obtiveram valores inferiores ou iguais ao valor geral de alinhamento foram destacadas as questões que impactaram tal resultado e analisadas individualmente. Lima (2008) propõe que as questões que obtiveram valores diferentes do valor geral, esses devem ser comparados aos desejados pela organização e a partir disso traçar planos para obter um 
aumento no valor do alinhamento. A Tabela 2 demonstra os valores observados.

No atributo comunicação, o nível do alinhamento atual é três (3). Com base nas respostas que apresentaram valor dois (2), para a melhoria do nível de maturidade propõe-se um maior compartilhamento do conhecimento da área da TI quanto à utilização dos indicadores dos setores operacionais, visto que os respondentes perceberam que tais informações estão restritas à área da TI. De acordo com o modelo de Lima (2008), para atingir o nível cinco (5) é preciso que a organização compartilhe as informações tanto interna quanto externamente, além de promover um melhor relacionamento entre a área da TI e os demais setores da organização. O atributo valor como resultado do nível de alinhamento atual é três (3) com apenas uma questão nota dois (2), que trata do acesso aos indicadores de desempenho do setor, os quais são percebidos apenas em nível funcional. Para o aumento do nível propõe-se que a TI atue de forma a promover o acesso dos gestores dos outros setores aos indicadores tanto da área de cada um quanto da organização.

$\mathrm{O}$ atributo habilidades obteve como nota do alinhamento um três (3), porém a questão que trata da gestão da inovação por parte da TI obteve nota dois (2), que sugere uma melhoria quanto à atuação inovadora da área da TI, além de uma visão comercial apoiando a gestão com projetos inovadores que permitam o acesso às informações necessárias para tomada de decisão. 
Tabela 2. Resultado da análise do alinhamento estratégico entre a área da TI e o negócio

\begin{tabular}{|c|c|c|c|}
\hline Atributos & Valor Atual & $\begin{array}{cc}\text { Nível } & \text { Nível } \\
\text { Alinhamento } & \text { Alinhamento Valor Desejado } \\
\text { Atual } & \text { Desejado }\end{array}$ & $\begin{array}{l}\text { Oportunidades de } \\
\text { melhoria }\end{array}$ \\
\hline
\end{tabular}

\section{COMUNICAÇ̃̃O}

Como a área de TI e seu

departamento compartilham

conhecimento?

Há algumas práticas, não

formalizadas, de compartilhamento

de conhecimento, como oficinas de trabalho e treinamentos.
A organização compartilha conhecimento internamente e também externamente, com

fornecedores, clientes e usuários.
Projeto Indicadores de Desempenho - BI
Como é percebida a atuação conjunta entre as reas de TI e de seu departamento?
Há atuação conjunta, mas limitada ao nível tático.
Além do reconhecimento interno da eficácia do relacionamento entre o departamento e a área de TI, há, também, uma percepção externa, dos fornecedores, clientes e usuários.

\section{VALOR}

$\begin{array}{ll}\text { Na sua opinião, os } & \text { são relatadas somente para o } \\ \text { indicadores do seu } & \text { pessoal de TI alocado no mesmo } \\ \text { departamento: } & \text { nível funcional. }\end{array}$

além de medir o valor agregado da TI, são estendidas a parceiros externos.
Projeto Indicadores de Desempenho - BI

\section{HABILIDADES}

Na sua visão, como a área de TI é incentivada a atuar com uma postura comercial e de inovação?

Algumas áreas de negócio incentivam a TI a atuar com uma postura comercial e de inovação.
5

O incentivo à atuação da TI com uma postura comercial e de inovação é normatizado e reconhecido por toda a organização.
Projeto Indicadores de Desempenho - BI

Fonte: do próprio autor 
Baseado na análise dos atributos e no confronto com os dados coletados dos relatórios de planejamento de cada setor, observou-se a necessidade de criação de uma ferramenta de BI, corroborando com o que foi observado por Lima (2008) quando os gaps gerados com as análises do resultado do cálculo do nível de alinhamento representam oportunidades de otimização dos processos por meios do uso eficiente dos recursos da TI. Luftman et al (2017) também observaram que os resultados da avaliação do nível de alinhamento podem ser utilizados para articular planos de ação mais efetivos nas áreas que garantam que a TI está sendo mais bem aproveitada para agregar valor ao negócio.

Neste estudo, o objetivo é demonstrar a implantação de uma plataforma de um BI, que foi uma das oportunidades de melhoria detectada por meio da análise do alinhamento.

\subsection{Implantação do BI}

Nesta etapa foi realizada análise documental dos resultados obtidos da análise do alinhamento entre a TI e o negócio observou-se que os acessos às informações por meio dos recursos da TI não eram utilizados pelas áreas do negócio de forma consolidada e ágil, o que dificulta o processo de tomada de decisão. Também foi constatando que estas áreas também demandavam informações para construir as suas estratégias que até então eram baseadas em suas rotinas, quando as informações estavam dispersas em seus sistemas e restritas à expertise e ao conhecimento de mercado.

A implantação do BI foi iniciada com a definição dos indicadores e das bases de dados. Para garantir a confiabilidade e integridade das informações, a diretoria da empresa determinou que a Controladoria atuasse juntamente com a área da TI na construção dos indicadores de cada setor.

Para a definição dos indicadores, foram realizadas entrevistas com a diretoria da empresa, onde foi observado que a organização já possuía 
indicadores de acordo com os objetivos estratégicos da empresa, agrupados por setor e empresa. A Tabela 3 apresenta os setores e indicadores.

Tabela 3. Relação de indicadores por setor

\begin{tabular}{ll}
\hline Setor & Indicadores \\
\hline Vendas & \\
- Veículos Novos & Faturamento, retorno F\&I, emplacamentos / transferências, \\
- Veículos Usados & lucro bruto, despesas fixas, despesas semi-fixas, estoque. \\
- Venda Direta & \\
\hline Pós-Vendas & Faturamento, lucro bruto, despesas fixas, despesas semi-fixas, \\
- Peças & estoque, OS abertas, OS com venda pendente, OS com reserva \\
- Serviços & de peças.
\end{tabular}

Fonte: do próprio autor.

Em seguida, a Controladoria mapeou a origem dos dados para a construção dos indicadores e observou que os controles eram realizados por meio de planilhas eletrônicas dispersas em várias áreas da empresa. Também foi constatado que não haviam relatórios com os informativos diários para acompanhamento das transações realizadas (vendas, compras, estoques) e das metas estabelecidas, visto que as informações eram enviadas por meio do fechamento contábil (Demonstrativo de Resultado do Exercício) que demandava alguns dias para que as informações fossem consolidadas. Os dados das planilhas eram basicamente provenientes do sistema de gestão da empresa, porém dispersos em várias telas, prejudicando a visualização da informação.

Nesse processo, várias rotinas foram otimizadas e identificados pontos a melhorar, tanto na oferta da informação quanto na disponibilização de informações confiáveis e tempestivas.

A partir da identificação das fontes de dados dos indicadores, a área da TI construiu relatórios com os campos do banco de dados do sistema de gestão utilizado pela empresa. A Controladoria realizou a validação dos 
dados e a formatação das informações de acordo com a necessidade de cada área. A Tabela 4 apresenta o modelo da base de dados da área de peças.

Tabela 4. Base de dados para elaboração do Painel de Indicadores

\begin{tabular}{|c|c|c|}
\hline Faturamento & Campos & Descrição \\
\hline \multirow{7}{*}{$\begin{array}{l}\text { Orçamento / Pré-Notas } \\
\text { Pendentes }\end{array}$} & Venda & Valor de Venda de Peças \\
\hline & Margem & $\%$ Margem de Venda = Venda / Custo Contábil \\
\hline & Lucro Bruto & Venda - Custo \\
\hline & Devoluções & Valor Devolução de Venda \\
\hline & Qt. Pedido & Quantidade de Orçamentos e Pré-Vendas \\
\hline & Qt. Itens & Quantidade de Itens \\
\hline & Valor & Valor de Venda de Peças \\
\hline \multirow[t]{2}{*}{ Oficina (OS) } & Vr. Peças & \\
\hline & Vr. Serviços & \\
\hline Pendências Anomalias & & Valor Informado na Geração do Relatório \\
\hline Pendências Garantia & & Valor Informado na Geração do Relatório \\
\hline \multirow[t]{5}{*}{ Es toque } & Campos & Descrição \\
\hline & Quant. & Quantidade de Itens \\
\hline & Reposição & Valor de Reposição \\
\hline & Contábil & Valor Custo Contábil \\
\hline & Venda & Valor de Venda \\
\hline \multirow[t]{4}{*}{ Peças Obsoletas 180 dias } & Quant. & Quantidade de Itens \\
\hline & Reposição & Valor de Reposição \\
\hline & Contábil & Valor Custo Contábil \\
\hline & Venda & Valor de Venda \\
\hline \multirow[t]{2}{*}{ Reserva de Peças (itens) } & Quant. & Quantidade de Itens \\
\hline & Valor & Valor de Venda \\
\hline $\begin{array}{l}\text { Giro Estoque (Venda/Estoque } \\
\text { Médio) }\end{array}$ & Valor & $\begin{array}{l}\text { Valor Cus to Contábil de Venda / (Estoque Inicial - Estoque } \\
\text { Final mês Contábil) }\end{array}$ \\
\hline $\begin{array}{l}\text { Rotatividade Estoque } \\
\text { (Vendas/Estoque) }\end{array}$ & Valor & $\begin{array}{l}\text { Valor Cus to Contábil de Venda / (Estoque Atual - Custo } \\
\text { Contábil) }\end{array}$ \\
\hline Campanhas e Objetivos & Campos & Descrição \\
\hline \multirow[t]{2}{*}{ Campanhas de Venda } & Campanha A & Valor Informado na Geração do Relatório \\
\hline & Campanha B & Valor Informado na Geração do Relatório \\
\hline \multirow[t]{2}{*}{ Objetivos de Compra } & Compra B & Valor Informado na Geração do Relatório \\
\hline & Bônus & Valor Informado na Geração do Relatório \\
\hline \multicolumn{3}{|l|}{ Clientes Especiais } \\
\hline \multirow{2}{*}{$\begin{array}{l}\text { Bloqueados com Venda no } \\
\text { Período }\end{array}$} & Campos & Descrição \\
\hline & Total NF & $\begin{array}{l}\text { Valor de NF vendas realizadas para clientes bloqueados no } \\
\text { período informado }\end{array}$ \\
\hline \multirow[t]{4}{*}{ Despesas DRE } & Campos & Descrição \\
\hline & Valor da & \\
\hline & Despesa & Valor da Despesa Contábil \\
\hline & Mensal & \\
\hline
\end{tabular}

Fonte: do próprio autor. 
Essa etapa evidenciou, conforme Reginato e Nascimento (2007), a importância da atuação da Controladoria juntamente com a área da TI quanto à confiabilidade das informações mediante a validação das bases de dados utilizadas para a consolidação dos indicadores.

Os relatórios gerados integraram informações de diversas bases de dados e possibilitaram a área da TI conhecer toda a estrutura do banco de dados para a construção do BI. A Tabela 5 demonstra um trecho do relatório final do setor de peças.

Tabela 5. Painel de Indicadores - Peças

\section{Painel de Indicadores}

Período: 01/01/20x8 a 31/01/20x8

\begin{tabular}{|c|c|c|c|c|c|c|c|}
\hline \multicolumn{3}{|c|}{ Faturamento } & \multicolumn{5}{|c|}{ Estoque } \\
\hline & Venda & Margem & & Qtd. & Reposição & Contábil & Venda \\
\hline Tele-Peças & $87.509,58$ & $100,0 \%$ & Peça X & 12 & $95.668,82$ & $81.892,16$ & $173.320,21$ \\
\hline Peça X & $5.096,47$ & $5,3 \%$ & Acess. Tipo X & 32 & $94.222,55$ & $98.650,39$ & $165.483,41$ \\
\hline Lub. Tipo X & $16.063,22$ & $14,5 \%$ & Acess. Tipo Y & 8 & $6.316,04$ & $6.200,17$ & $13.380,19$ \\
\hline Lub. Tipo Y & $20.397,78$ & $16,1 \%$ & Lub. Tipo X & 1 & 165,46 & 165,42 & 459,65 \\
\hline Peças Tipo X & $11.033,17$ & $14,2 \%$ & Lub. Tipo Y & 8 & $2.360 .433,65$ & $684.745,03$ & $11.197 .438,89$ \\
\hline Peças Tipo Y & $34.918,93$ & $50,0 \%$ & Peças Tipo X & 205 & $1.391 .005,60$ & $487.904,05$ & 2.714.577,04 \\
\hline \multirow[t]{3}{*}{ Sub-Total } & $87.509,58$ & & Peças Tipo XY & 303 & $2.484 .285,05$ & $1.392 .762,14$ & $4.744 .236,11$ \\
\hline & & & Peças Tipo Y & 3 & $1.761,46$ & $1.761,45$ & $3.532,75$ \\
\hline & Venda & Margem & Pneus/Cam. & 8 & $64.029,46$ & $62.163,53$ & $93.872,32$ \\
\hline Acessórios & $7.034,76$ & $5,1 \%$ & & 0 & 0,00 & 0,00 & 0,00 \\
\hline Acess. Tipo X & $5.806,89$ & $87,0 \%$ & Peça X & 37 & $5.168,80$ & $4.613,85$ & $9.527,91$ \\
\hline Acess. Tipo Y & $1.179,36$ & $12,6 \%$ & Acess. Tipo X & 88 & $5.371,74$ & $4.807,49$ & $9.718,89$ \\
\hline Peças Tipo X & 48,51 & $0,3 \%$ & Acess. Tipo Y & 76 & 274,59 & 274,62 & $1.183,01$ \\
\hline Funilaria/Pint. & 149,66 & $0,2 \%$ & Loja & 0 & 0,00 & 0,00 & 0,00 \\
\hline Lub. Tipo X & 51,63 & $36,8 \%$ & Kits & 27 & 0,00 & 0,00 & 0,00 \\
\hline Lub. Tipo Y & 50,68 & $34,4 \%$ & Lub. Tipo X & 2 & 0,00 & 0,00 & 0,00 \\
\hline Peças Tipo X & 15,89 & $9,7 \%$ & Lub. Tipo Y & 7 & $26.021,60$ & $6.689,70$ & $19.770,81$ \\
\hline Peças Tipo XY & 5,90 & $3,7 \%$ & Mat. Tipo X & 0 & 1,53 & 1,55 & 5,47 \\
\hline Peças Tipo Y & 25,56 & $15,4 \%$ & Outras Merc. & 5 & 71,96 & 71,97 & 199,41 \\
\hline Mecânica & $95.564,74$ & $94,6 \%$ & Peças Tipo X & 619 & $48.784,85$ & $35.978,25$ & $84.520,58$ \\
\hline Peça X & $13.610,96$ & $13,8 \%$ & Peças Tipo XY & 140 & $16.411,99$ & $12.866,04$ & $29.794,60$ \\
\hline Acess. Tipo Y & 32,59 & $0,1 \%$ & Peças Tipo Y & 280 & 4.697,08 & $4.580,12$ & $12.701,99$ \\
\hline Loja & 44,37 & $0,0 \%$ & Pneus/Cam. & 10 & $7.443,92$ & $7.330,61$ & $11.571,24$ \\
\hline Lub. Tipo X & $12.369,36$ & $16,3 \%$ & & 1.871 & $14.500 .298,60$ & $6.345 .303,83$ & $42.292 .312,65$ \\
\hline Lub. Tipo Y & $12.056,06$ & $17,3 \%$ & & & & & \\
\hline Outras & 3,31 & $0,0 \%$ & & & & & \\
\hline Peças Tipo X & $23.397,76$ & $18,5 \%$ & & & & & \\
\hline Peças Tipo XY & $15.630,35$ & $15,2 \%$ & & & & & \\
\hline Peças Tipo Y & $11.959,12$ & $15,8 \%$ & & & & & \\
\hline Pneus/Cam. & $6.460,87$ & $3,1 \%$ & & & & & \\
\hline
\end{tabular}

Fonte: do próprio autor. 
Para a construção do BI, a empresa adotou uma solução com recursos gráficos por meio de dashboards (Figura 4) e que também permite acesso aos dados em tempo real de forma personalizada por área. A plataforma do BI também engloba soluções de acesso por aplicativos que garantem maior mobilidade.

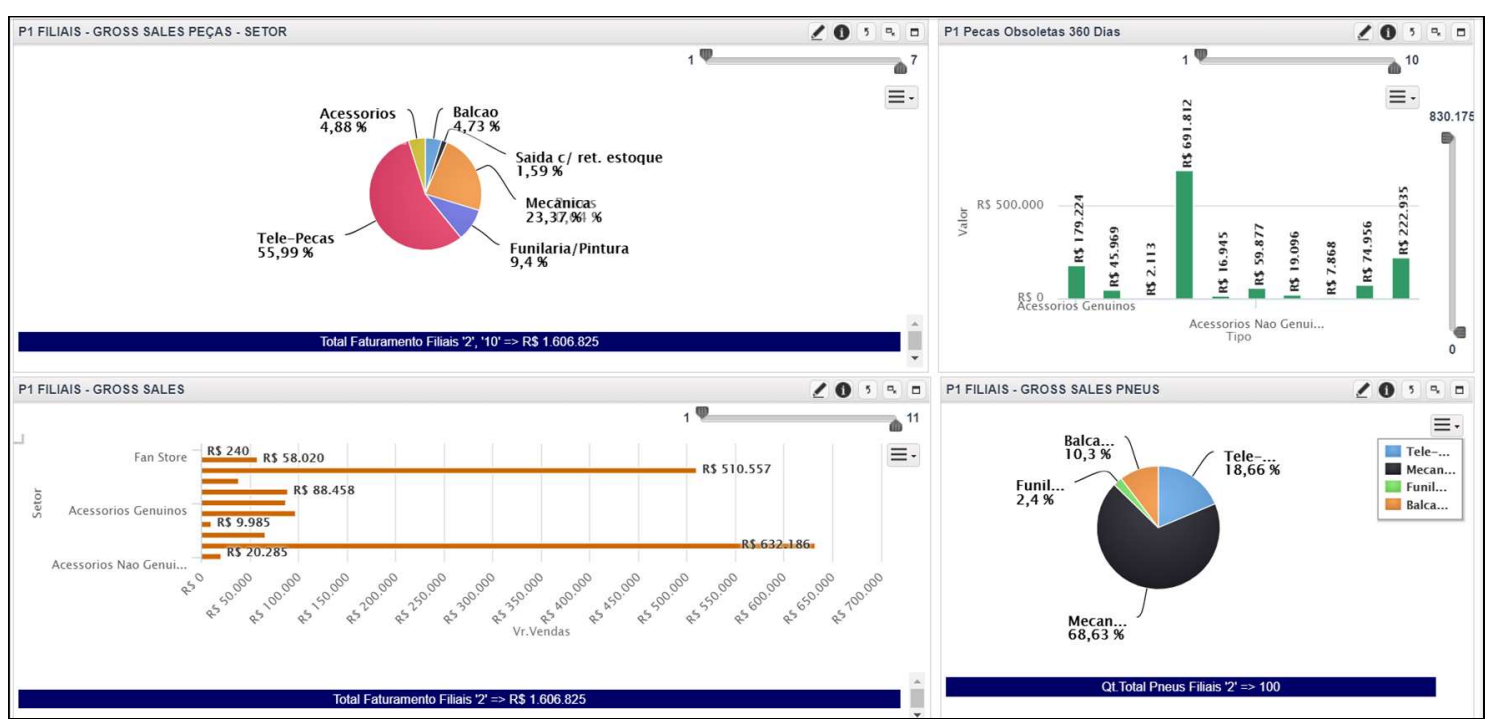

Figura 4. Dashboard Peças Fonte: do próprio autor.

O BI supriu as expectativas iniciais do projeto de sua implantação na empresa estudada, pois permitiu a visualização dos indicadores de desempenho de forma gráfica e intuitiva em uma única tela, viabilizando a melhor utilização dos recursos da TI da organização para melhoria do nível de alinhamento entre a TI e o negócio.

Tal constatação também foi verificada por Strate e Pretto (2015) que destacaram a importância da visualização rápida dos aspectos chaves de um negócio com o uso de dashboards. Fraga, Erpen, Varvakis e Santos (2017) constataram flexibilidade e agilidade no processo da tomada de decisão com o uso de dashboards. 


\section{Considerações Finais}

No ambiente atual, o papel da tecnologia da informação (TI) é fundamental para as organizações crescerem e sobreviverem no mercado. Entretanto, essas devem buscar maneiras para que a tecnologia possa viabilizar o seu sucesso.

Este estudo demonstrou a implantação de um sistema de BI a partir do diagnóstico do nível de alinhamento entre a TI e o negócio. Verificou-se que a análise do nível de alinhamento propiciou uma melhor visão do nível atual da organização e as oportunidades de melhoria, possibilitando que os recursos da TI sejam utilizados de forma efetiva pela organização. Os resultados obtidos corroboram com os resultados encontrados por outros autores que também comprovaram que o sucesso da organização poderá ser obtido por meio do alinhamento entre a TI e o negócio (Affeldt et al 2006; Joia e Souza 2009; Amarilli et al 2017).

Constatou-se também que o apoio da Controladoria na definição dos indicadores garantiu a integridade e confiabilidade das informações, de acordo com as necessidades dos gestores da organização. Essa ação, de acordo com Luftman, Lyytinen e Zv1 (2017), confirma a importância da integração entre as áreas funcionais da empresa e a TI para a promoção de um maior alinhamento com o negócio.

Espera-se, com este trabalho, como contribuição teórica, incrementar os estudos referentes à implantação do BI em empresas com a adoção do diagnóstico do nível de maturidade entre a TI e o negócio. Como contribuição para o mercado, este estudo proporcionará uma evidenciação de um caso de implantação do BI em empresas do ramo de concessionárias de veículos no interior de Minas Gerais.

Como sugestão de futuros estudos, apontamos que a metodologia da análise da maturidade seja aplicada após a implantação do BI para evidenciar o aumento do nível de maturidade. 


\section{Referências}

Affeldt, F. S., Silveira, F. C. D. S., \& Vanti, A. A. (2006). Análise Trinômio Alinhamento Estratégico x Business Intelligence (BI) x Controladoria Estratégica. In: Anais do XIII Congresso Brasileiro de Custos - Belo Horizonte -MG, Brasil, 30.

Amarilli, F., Van Vliet, M., \& Van Den Hooff, B. (2017 - Dezembro). An Explanatory Study on the Co-Evolutionary Mechanisms of Business IT Alignment. In 38th International Conference on Information Systems: Transforming Society with Digital Innovation, ICIS 2017, Seoul, South Korea,10 (pp. 1-22). AES Electronic Library.

Bezerra, A. A., \& Siebra, S. A. (2015). Implantação e Uso de Business Intelligence: Um Relato de Experiência no Grupo Provider. Revista Eletrônica de Gestão Organizacional, 13(3), 233-243.

Barbieri, C. (2011). BI2--Business Intelligence: Modelagem \& Qualidade. Elsevier Editora.

Demo, P. (2009). Metodologia do Conhecimento Cientifico. 7. reimpressão. São Paulo: Atlas.

Fatima, A., \& Linnes, C. (2019). The Current Status of Business Intelligence: A Systematic Literature Review. American Journal of Information Technology, 9(1), 1-22.

Federação Nacional da Distribuição de Veículos Automotores. (2021). Anuário 2020 O desempenho da distribuição automotiva no Brasil. Recuperado em 05 março, 2021, de http://www.fenabrave.org.br/portal.

Fraga, B. D., Erpen, J. G., Varvakis, G., \& dos Santos, N. (2017). Business Intelligence: métodos e técnicas de gestão do conhecimento e as tendências para avanços do capital intelectual. Navus-Revista de Gestão e Tecnologia, 7(1), 43-56. http://dx.doi.org/10.22279/navus.2017.v7n1.p43-56.410

Gil, A. C. Como Elaborar Projetos de Pesquisa. 5 ed. São Paulo: Atlas, 2010.

Guarda, T., Pinto, F. M., Cordova, J. P., Mato, F., Quiña, G. N., \& Augusto, M. F. (2016, June). Pervasive Business Intelligence as a Competitive Advantage. In: 11th Iberian Conference on Information Systems and Technologies (CISTI), 2016, Canary Islands, Spain, 15 (pp. 1-4). IEEE.

Johnson, G., Scholes, K., \& Whittington, R. (2011). Fundamentos de Estratégia. Bookman Editora. 
Joia, L. A., \& Souza, J. G. A. D. (2009). Articulando Modelos de Alinhamento Estratégico de Tecnologia da Informação. Cadernos EBAPE.BR, 7(2), 252269. https://doi.org/10.1590/S1679-39512009000200006

Kenneth, C. L., \& Laudon, J. P. (2011). Sistemas de Informação Gerenciais. Editora Person. São Paulo.

Lima, A. G. B. (2008). Diretrizes para um processo de diagnóstico da governança de tecnologia da informação. (Dissertação de Mestrado, Universidade Católica de Brasilia). Brasília, DF, Brasil.

Luftman, J. (2004). Assessing Business-IT Allignment Maturity. In W. Van Grembergen (Eds.), Strategies for Information Technology Governance (pp. 99-128). IGI Global. http://doi:10.4018/978-1-59140-140-7.ch004

Luftman, J., Lyytinen, K., \& Zvi, T. B. (2017). Enhancing the measurement of information technology (IT) business alignment and its influence on company performance. Journal of Information Technology, 32(1), 26-46. https://doi.org/10.1057/jit.2015.23

Moreira Pereira, O., Pereira Zaupa, A., Virginio Maracci, F., \& Bocchi Pedroso, C. (2016, April). Proposta para Implantação de Business Intelligence em Shopping Center. Colloquium Exactarum, 8(2), 13-26. http://dx.doi.org/10.5747/ce.2016.v08.n2.e152

Pinto, S. A. O. (2012). Estruturas de Gestão de Informações para Inteligência de Negócio nas Organizações e o Impacto Individual nas Atividades (Tese de Doutorado, Faculdade de Economia, Administração e Contabilidade / Universidade de São Paulo). http://dx.doi.org/10.11606/T.12.2012.tde$\underline{05112012-183755}$

Reginato, L., \& Nascimento, A. M. (2007). Um Estudo de Caso Envolvendo Business Intelligence como Instrumento de Apoio à Controladoria. Revista Contabilidade \& Finanças-USP, 18, 69-83. https://doi.org/10.1590/S1519$\underline{70772007000300007}$

Rezende, D. A., \& Abreu, A. F. D. (2000). Tecnologia da Informação Aplicada a Sistemas de Informação Empresariais. São Paulo: Atlas.

Serra Costa, J. F., \& Duarte, K. S. (2010). Escolha da Ferramenta Adequada para o Desenvolvimento de Painéis de Indicadores em uma Empresa de Seguros: uma Abordagem Multicritério. Sistemas \& Gestão, 5(1), 32-49. https://doi.org/10.7177/sg.2010.V5N1A3

Silva, R. A., Silva, F. C. A., \& Gomes, C. F. S. (2016). O uso do Business Intelligence (BI) em Sistema de Apoio à Tomada de Decisão Estratégica. 
Revista GEINTEC-Gestão, Inovação e Tecnologias, 6(1), 2780-2798.

https://doi.org/10.47059/geintecmagazine.v6i1.726

Silveira, V. N. S. (2017). Alinhamento estratégico: Análise Conceitual e Perspectivas Teóricas. Revista Pretexto, 18(3), 67-91.

https://doi.org/10.21714/pretexto.v18i3.5675

Sharda, R., Delen, D., \& Turban, E. (2009). Business Intelligence e Análise de Dados para Gestão do Negócio. 4. Ed. Bookman Editora.

Strate, L. H., \& Pretto, F. (2015). Gestão de TI: Implantação de um Software de Chamados Técnicos e Geração de Indicadores de BI. Revista Destaques Acadêmicos, 7(1), 150-162.

Watson, H. J., \& Wixom, B. H. (2007). The Current State of Business Intelligence. Computer, 40(9), 96-99. https://doi.org/10.1109/MC.2007.331 